
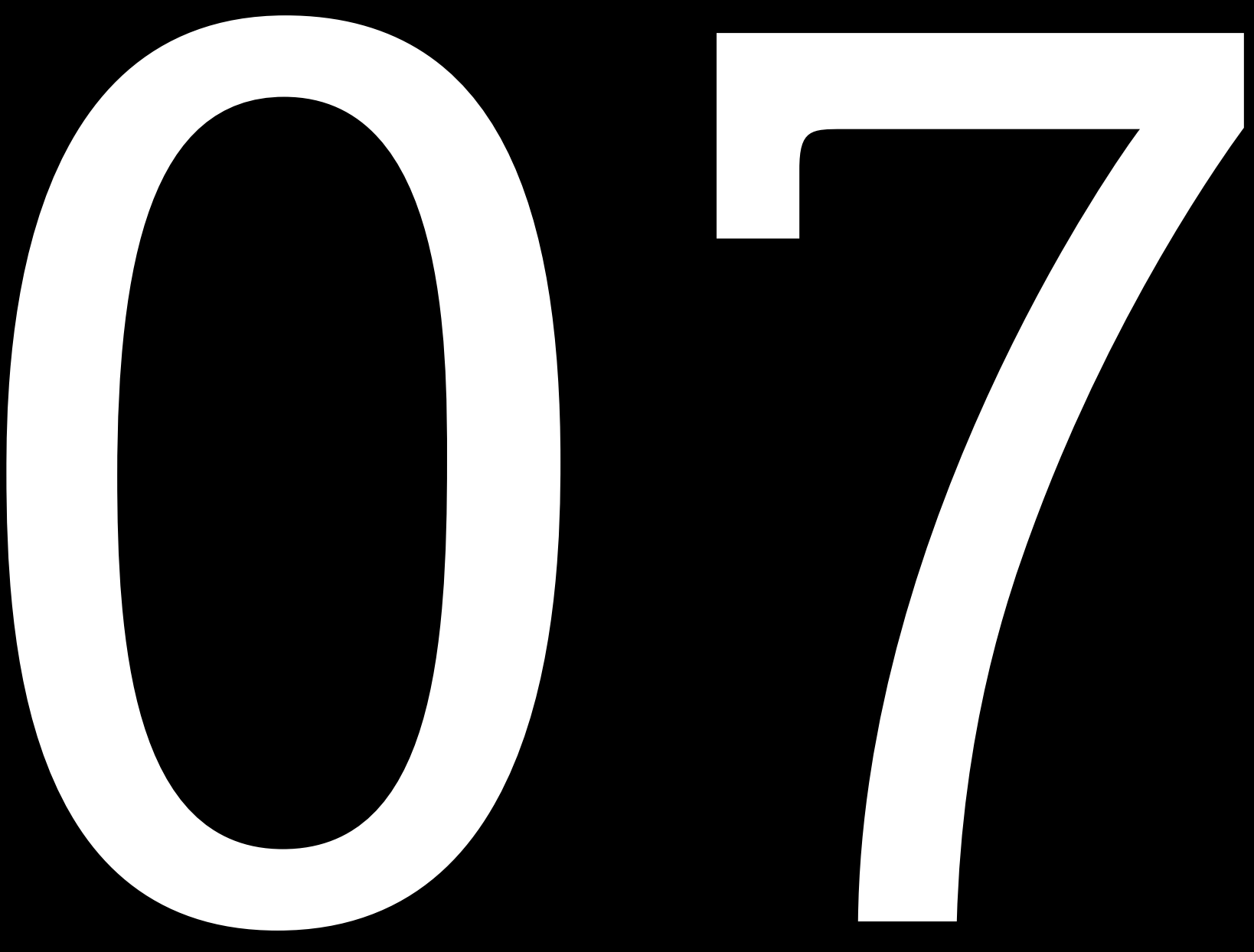

DOI: https://doi.org/10.14483/2422278X.14762 


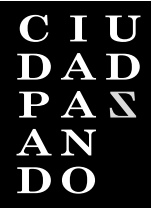

\section{Enfoque diferencial en el marco de la Ley 1448 de 2011 en el municipio de Cajicá- Colombia $^{1}$}

Differential approach within Law 1448 of 2011 framework in the Cajicá municipality, Colombia

\section{Laity Alvinzy Velásquez Fandiño ${ }^{2}$, Luisa María Bernal Ruiz ${ }^{3}$}

Para citar este artículo: Velásquez, L. y Bernal, L. (2020). Enfoque diferencial en el marco de la Ley 1448 de 2011 en el municipio de Cajicá-Colombia. Ciudad Paz-ando, 13(1), pp. 81-92.

Fecha de recepción: 9 de abril de 2019

Fecha de aprobación: 18 de mayo de 2020

1 Artículo de investigación producto del Proyecto INVDIS 2567 financiado por la Vicerrectoría de Investigaciones de la Universidad Militar Nueva Granada para la vigencia 2018.

2 Docente Titular, Universidad Militar Nueva Granada. Doctor en Bioética, Universidad El Bosque. Correo electrónico: laity.velasquez@ unimilitar.edu.co

3 Antropóloga, Pontificia Universidad Javeriana. Asistente de Investigación, Universidad Militar Nueva Granada. Correo electrónico: lubernalr@gmail.com 


\section{RESUMEN}

La situación del desplazamiento forzado producto del conflicto armado en Colombia es abordada por la Ley 1448 del 2011 con un enfoque llamado diferencial. Con base en la ley, este artículo pretende responder la siguiente inquietud ¿cómo el municipio de Cajicá brinda asistencia a las víctimas aplicando el enfoque diferencial? La investigación abordó la problemática desde la Evaluación realista, en un proceso de formulación y refinamiento sucesivo de hipótesis, con técnicas de recolección de información entrevistas semiestructuradas, que permitieron el replanteamiento hipotético. Los resultados evidencian que no existe un enfoque diferencial en el municipio. Los factores ahondados en el artículo muestran este aspecto y permiten evidenciar que la relación entre funcionarios y víctimas es una relación asistencialista. Dado que no hay una caracterización de las víctimas las ofertas se generalizan, se están brindado ayudas sectorizadas, existe desarticulación tanto vertical como horizontal, así como una escaza asignación presupuestal.

Palabras clave: aplicación Ley 1448/2011, desplazamiento, política pública, víctima del conflicto armado.

The forced displacement situation product of armed conflict in Colombia, is approached by the Law 1448 of 2011 with a focus called differential. Based on this law, this article aims to respond the next inquiry ¿How is the municipality of Cajica giving assistance to the victims applying the differential focus? The investigation approached the troublesome since realistic evaluation, in a formulation and refinement process following the hypothesis, with information recollection techniques, semi-structured interviews, that allowed the hypothetic rethinking. The results evidence there is no such thing as differential focus on the municipality. The in-depth factors in the article are showing this aspect and allow the evidence that the relationship between officials and victims is a welfare relationship. Since there is no characterization of the victims, offers are generalized, sectorized assistance is being given, there is both vertical and horizontal disarticulation, as well as a lack of budget allocation.

Keywords: Law 1448/2011 application, displacement, public politic, armed conflict victim. 


\section{Introducción}

Según el Informe Basta ya. Memoria de guerra y dignidad, elaborado por el CNMH (2013), del conjunto de muertes violentas ocurridas en el país entre 1958 y 2012, por lo menos 220000 tienen su origen en el conflicto armado y, de estas, el $80 \%$ han sido civiles inermes. Se trata de cientos de miles de víctimas producto, en especial, de masacres y asesinatos selectivos, a las que hay que sumar muchos otros miles de víctimas de desaparición forzada, desplazamiento forzoso, secuestros, ejecuciones extrajudiciales, reclutamiento ilícito, tortura y sevicia, minas antipersonales y violencia sexual (CNMH, 2013).

En cuanto al desplazamiento forzado, según el CNMH (2013), "se estima que por lo menos 8.3 millones de hectáreas y 350.000 predios fueron abandonados y despojados durante los peores años del conflicto, entre 1996 y el 2002" (p. 28). Para el caso de Cajicá, de acuerdo con el Informe de atención a víctimas del conflicto armado residentes en el municipio de Cajicá, durante los años 2013, 2014 y 2015 aumentó la cantidad de hogares que llegaron a este municipio. Por otro lado, de acuerdo con el reporte del registro único de víctimas (RUV), en el 2019 residen 1233 personas.

A primera vista, las cifras generalizan la violencia; no obstante, es importante leer esos números reconociendo que la violencia ha impactado diferencialmente a la población en razón de las categorías de discapacidad, grupos étnicos, género, orientación sexual e identidad de género, y rango etario.

El siguiente artículo procede del estudio de caso realizado por el Proyecto INVDIS 2567 financiado por la Vicerrectoría de Investigaciones de la Universidad Militar Nueva Granada para la vigencia 2018, con un grupo de víctimas del conflicto armado en residentes el Municipio de Cajicá. A través del mismo, se pretende analizar el tipo de asistencia que recibe esta población, de tal modo que se pueda identificar la forma en que es garantizada la reparación integral, asistencia y atención, de acuerdo con el enfoque diferencial, artículo 13 de la Ley de víctimas 1448 de 2011.

Asimismo, se busca trazar un abordaje metodológico para analizar y discutir dentro del ámbito académico la manera como se ejecuta la política pública sobre víctimas, ello a través de lo que puede aportar el estudio de caso dentro del contexto del municipio de Cajicá- Colombia.

\section{Enfoque metodológico}

Con miras a evaluar políticas públicas, existen diferentes ejes teóricos para dimensionar la magnitud del efecto de un programa, también para efectuar orientaciones sobre la calidad de las políticas públicas y pensar en soluciones prácticas para atender las necesidades apremiantes del país, al igual que generar recomendaciones que incrementen la eficacia y la eficiencia de las acciones de un gobierno.
Pawson y Tilley (1997), Westhorp (2014) y Parra (2017), entre otros, han trabajado con el eje teórico de la evaluación realista (ER). En lo tocante al eje, señalan la importante de precisar la diferencia entre este tipo de evaluación y la de evaluación de impacto (EI).

En el Manual para la evaluación de impacto de proyectos y programas de lucha contra la pobreza, Navarro (2005), citando diferentes autores, define la EI como la medición de los cambios en el bienestar de los individuos que pueden ser atribuidos a un programa o a una política específica. Su propósito general es determinar la efectividad de las políticas, programas o proyectos ejecutados. Al igual que otras técnicas de evaluación sumativa, la EI se puede utilizar para determinar hasta qué punto los resultados planificados fueron producidos o logrados, así como para mejorar otros proyectos o programas en ejecución o futuros (Navarro, 2005 p. 17).

En contraste con la EI, Parra (2017) y Westhorp (2014) aseguran que la ER abarca aspectos que la EI restringe; por ejemplo, puede responder en términos de los resultados ¿qué funciona?, ¿para quién?, ¿en qué aspectos?, ¿hasta qué punto?, ¿en qué contextos?, y ¿cómo?_Sobre la base de la anterior idea, Parra (2017) expone unos eslóganes para enunciar los principios de la evaluación de impacto y la realista. Frente al eslogan para la evaluación realista dice: "toda acción tiene un efecto, la pregunta es para quién y en qué contexto, o, toda hipótesis se alimenta de conocimiento previo (si se quiere, de un prejuicio inicial)" (Parra, 2017, p. 20).

En consideración con las diferencias entre estos dos tipos de evaluación, Westhorp (2014) señala que a través de la ER se puede captar una mejor comprensión de la efectividad de los programas a pesar de que no se alcance una certeza final.

En conformidad con Westhorp (2014), y siguiendo el pensamiento de Pawson y Tilley (1997), se afirma que para entender y evaluar los programas en la lógica de la ER hay que analizarlos como sistemas sociales abiertos. Así, particularmente se requiere:

una comprensión por lo menos general de la teoría de sistemas, una comprensión de los métodos apropiados para evaluar sistemas y una comprensión del sistema de programa específico. En segundo lugar, se necesita acotar el o los sistemas que se incluirán en la evaluación, aunque esos límites no existan claramente en la realidad. Tercero, esos sistemas no serán estáticos, sino que irán cambiando con el tiempo de maneras complejas e interactivas, ya sea que se pongan en juego programas y políticas o no. Tal vez lo más importante es que cualquier resultado observable será producto de interacciones al interior de los sistemas y entre ellos, no sólo el producto del programa o política. Cualquier resultado será producto de múltiples causas y cualquier acción o cambio en un sistema puede tener muchas consecuencias [Traducción propia]. (Westhorp, 2014, p. 4) 
Siguiendo la lógica de sistemas sociales abiertos es preciso que en el interior de los programas ocurran procesos inobservables que provocan un efecto (resultado) sobre determinada población que interactúa dentro de un contexto específico. Esos procesos inobservables son nombrados por los realistas como mecanismos. $\mathrm{Si}$ se apela a un ejemplo de lo inobservable, según Westhorp (2014) la gravedad es el modelo ideal. Westhorp afirma:

La idea es que las cosas que experimentamos o podemos observar tienen su origen en procesos más profundos, y usualmente imperceptibles. Así, por ejemplo, uno puede abrir la mano y observar la caída de la pelota de tenis que sostenía, pero no puede ver la gravedad que causa que la bola caiga [Traducción propia.]. (p. 5)

Por otro lado, teniendo en cuenta que los mecanismos son influenciados e interactúan con el contexto, es preciso decir que el entorno dentro del cual opera la política/programa puede influir en el desempeño de la misma, específicamente esto es lo que dice Westhorp (2014):

El contexto en que la organización implementa un programa puede influir en la manera o el alcance de la implementación, su público objetivo, alcances y demás. Además, puede influir en las respuestas que dan los potenciales beneficiarios. Las respuestas pueden diferir según si son agencias gubernamentales o no gubernamentales las que llevan a cabo el programa, por ejemplo, si la confianza en un sector es menor que en el otro. Las variaciones en el público objetivo (en género, casta, clase, cultura y demás) pueden determinar qué mecanismos operan, lo cual está a la base de la pregunta "para quién" en la evaluación realista. Tener acceso a los recursos y oportunidades para implementar decisiones puede influir sobre el mismo razonamiento, además de la posibilidad de poner en juego las alternativas deseables [Traducción propia]. (p. 6)

Más que referirse a locaciones geográficas (por ejemplo, un colegio) o temporales (por ejemplo, un colegio hace $\mathrm{X}$ años) per se, el contexto indaga por relaciones sociales, reglas formales e informales o disponibilidades de medios o de recursos materiales, pero también culturales, en las cuales entra a funcionar un proyecto, un programa o incluso una política social.

Resumiendo lo planteado, bien se puede inferir que, al entender los programas o las políticas públicas en la lógica de los sistemas sociales abiertos, se comprende el sentido de la ER. Del anterior sentido se deduce que, para evaluar los resultados de un programa, es posible entender el mismo en lógica de la elaboración de teorías organizadas por mecanismo, contextos, resultados, tres aspectos que los realistas abrevian como $\mathrm{CMO}^{4}$.

Técnicamente los CMO no deben ser enumerados como listas, ya que deben distribuirse de manera lógica como una cadena de sucesos, por ejemplo, en un contexto determinado, cierto mecanismo genera este resultado. Parra (2017) citando a Pawson ilustra el siguiente ejemplo textual que surge del contexto de un programa de generación de capacidades en una comunidad para atender las necesidades psicomotoras de niños y niñas en edad preescolar:

La estrategia de agrupar a niños y niñas con necesidades similares puede funcionar mejor para menores con dificultades lingüísticas y motoras finas (C), al permitir que estos miren y copien a otros para desarrollar sus propias habilidades motoras finas (M) y resultado en una mayor disposición para intentar nuevas actividades en el jardín de infancia y el hogar (O).

Para construir los mecanismos se necesita presentar unas teorías de rangos medios o preliminares (hipótesis). De acuerdo con Parra (2017), estas pueden encontrarse cifradas en el trabajo de otros investigadores, en la experiencia previa del investigador o en observaciones preliminares sobre el contexto en que ocurre el evento. Jagosh et al. (2015) afirma que las teorías de rango medio pueden ser usadas para enfocar las preguntas de investigación y desarrollar instrumentos de recolección. Igualmente, Parra (2017) menciona que, a través de las entrevistas o los grupos focales, se puede llegar a confirmar, falsear o refinar una teoría de rango medio.

La indagación de resultados por medio de teorías de rango medio posiciona de forma diferente al entrevistado; de esta forma, Parra (2017) afirma:

Las lógicas más clásicas de indagación en campo [...] tienden a asumir que el objetivo del despliegue de un instrumento (ej. un formulario de preguntas) es el entrevistado y no [...] la teoría del programa. Siendo ese el caso, el entrevistador no es un simple captador de información; el objetivo consiste en crear una situación en la cual los postulados/conceptos teóricos bajo investigación estén abiertos a la inspección. (p. 27)

La Figura 1 esclarece cómo es comprendida la evaluación realista en el marco del proyecto de investigación INVDIS 2567, el cual se referencia en el pie de página inicial. Esto implica reconocer qué es la evaluación realista, cuál es su lógica y cuáles son las hipótesis o teorías de rango medio que fueron base para la construcción de

4 Por su parte la nomenclatura: contextos (C), mecanismo (M), resultados $(\mathrm{O})$ abraca la abreviación original de sus términos en inglés. 


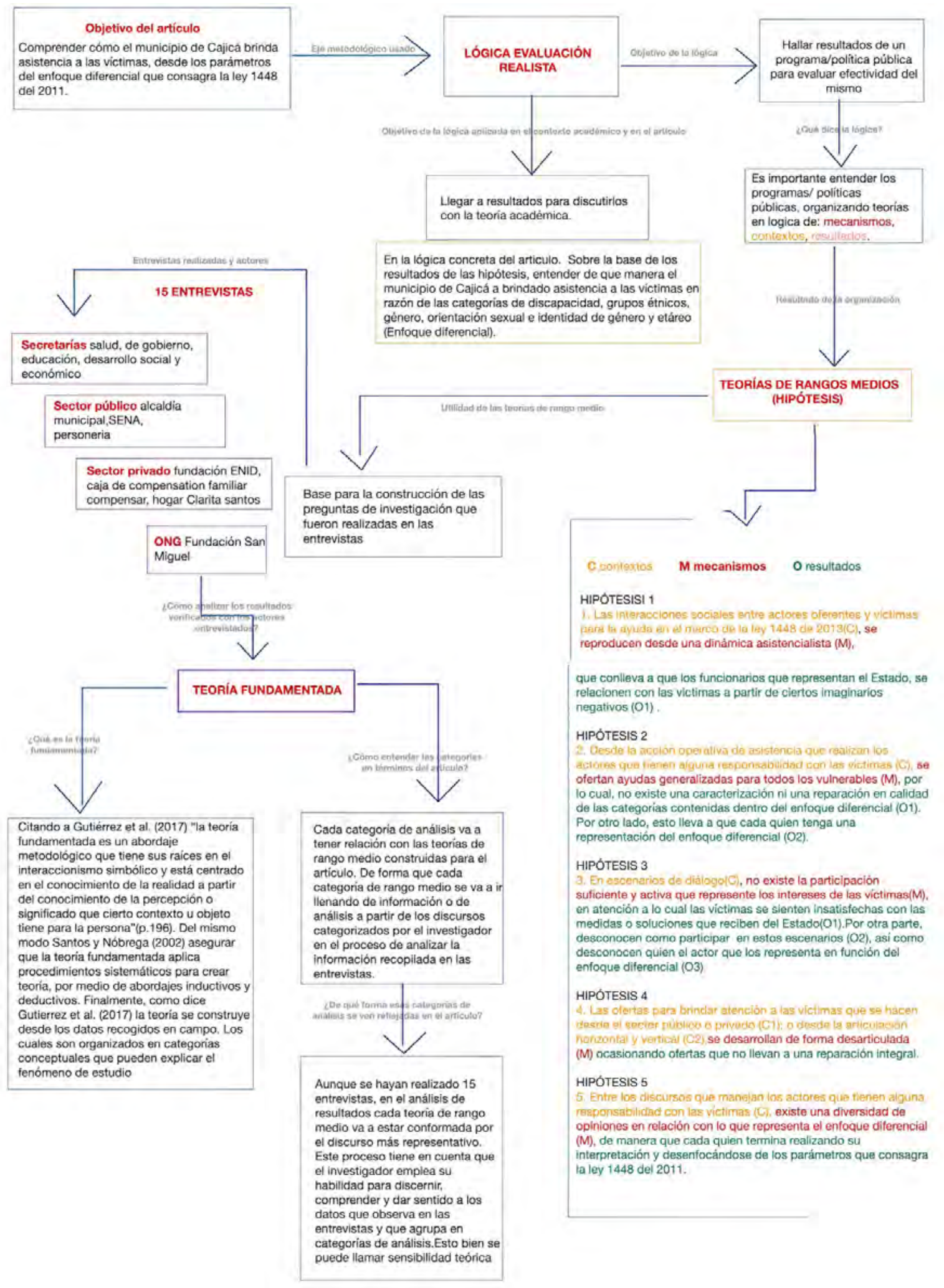

Figura 1. Proceso y procedimiento para la aplicación de la evaluación realista: estudio de caso de Cajicá Fuente: elaboración propia. 
las preguntas efectuadas en las entrevistas que se hicieron con quince actores del sector privado y público del municipio de Cajicá.

Simultáneamente, la Figura 1 abarca la estrategia y el eje metodológico (teoría fundamentada) que fue usado para analizar la información procedente de las entrevistas desarrolladas. Con ello se pretende cumplir con el objetivo planteado de mostrar el proceso metodológico que impla el uso de la evaluación realista aplicado a políticas públicas. Aunque se hayan realizado quince entrevistas para la corroboración de cada teoría de rango medio o hipótesis, los resultados de las mismas estarán sustentados con el discurso más representativo.

La lógica de la teoría fundamentada dice que través de los datos recogidos en campo se pueden construir teorías que expliquen las percepciones a través de las cuales las personas crean significados de su entorno. En términos de esta investigación, nos convocan las teorías que dan significado a las hipótesis y, por ende, a la comprensión de la aplicación del enfoque diferencial en el marco de la Ley 1448 de 2011. En la lógica de la teoría fundamentada, cada teoría puede estar organizada dentro de una categoría conceptual, la cual explicaría el fenómeno de estudio. En esta investigación, cada hipótesis es una teoría organizada en una categoría conceptual conformada por los diferentes discursos de quienes han sido entrevistados. Téngase en cuenta que la correspondencia mutua entre las opiniones de los entrevistados deriva un relato que explica o teoriza el significado de la hipótesis, brindando resultados que se pueden exponer a través de la voz de los entrevistados.

Teniendo en cuenta lo anterior, en la exposición de los resultados para cada hipótesis se expondrán los más representativos. Este proceso tiene en cuenta que el investigador emplea su habilidad para discernir, comprender y dar sentido a los que observa en las entrevistas y que agrupa en categorías de análisis.

\section{Análisis de información}

En este apartado se presentarán los resultados procedentes de las entrevistas realizadas en correlación con cinco hipótesis, tal como se describe en la Figura 1, anteriormente referenciada. Cabe señalar que los resultados que indican la hipótesis cuatro y cinco se relacionan con los resultados obtenidos en evoluciones externas a este estudio, tales como: (a) Evaluación de la política pública para la prevención de riesgos, la protección y garantía de los derechos de las mujeres víctimas del conflicto armado (Conpes 3784 de 2013); (b) Evaluación de operaciones y de resultados de la política de Retornos y Reubicación para el Departamento Nacional de Planeación (DNP). Lo anterior para expresar que existen congruencias tanto en lo analizado en el contexto de Cajicá como en los resultados de las investigaciones anteriormente señaladas.
Hipótesis 1. Las interacciones sociales entre actores ofertantes y víctimas para la ayuda en el marco de la Ley 1448 de 2013 se reproducen desde una dinámica asistencialista.

En los discursos de los actores oficiales y del sector privado del municipio, se observó que existe una diferenciación de estatus dada por el hecho de ser oriundo de Cajicá o no serlo y estar en la categoría de víctima. Analizando esta situación, es posible entender que, en esta división se establece una forma diferenciadora de apoyo. De cara a la posición que ocupan las víctimas, los funcionarios se relacionan con ellas considerándolas como sujetos especiales, indefensos, desprotegidos o con características culturales particulares que deben ser transformadas, lo que ocasiona una actitud compasiva o asistencialista. A continuación, se hará alusión a la forma en que el funcionario de una fundación ${ }^{5}$ representa a las víctimas:

la problemática social que se ha venido desarrollando y digamos en nuestra Sabana por la sobrepoblación, por la llegada de migrantes, digamos que nos hemos visto en la necesidad, porque realmente ya es una necesidad, debe ser establecido como una política pública el estar no solamente dispuestos, sino también el tener un plan para poder atender y formar a la población [...] sí, pues porque obviamente ya estamos hablando de un impacto social del impacto cultural de choques educativos porque vienen con una formación diferente, porque traen una cultura diferente y nosotros como institución debemos establecer un modelo pedagógico para poder atender a esta población, así como se encuentra en una condición desprotegido o vulnerable dado que ha tenido que resistirse al impacto. (Fundación, entrevista, 15 de diciembre del 2018)

Si bien existe en la representación que el funcionario hace de la víctima un tipo de subordinación al verlo como vulnerado, es importante destacar otro aspecto referente a la no enunciación de la persona dentro de la categoría de víctima, sino su referencia a través del uso indirecto de otro concepto. En las entrevistas siguientes se puede evidenciar este aspecto:

No deberíamos llamarlos a ellos víctimas [...], eso creo yo que es un error institucional llamarlos víctimas. Eso parece ser que en su autoestima los baja entonces yo soy víctima y estoy por debajo de todo el mundo porque soy víctima. Yo diría más bien llamarlas como héroe de guerra he soportado inclemencias de guerra dificultades me he

5 Téngase en cuenta que la identidad de los entrevistados será protegida, por tal razón las entrevistas no aludirán directamente a su nombre, así como tampoco se especificará la institución donde trabajan. 
perdido muchas cosas y he sido un sobreviviente de la guerra, soy un héroe. (Secretario de Desarrollo Económico, entrevista, 18 de diciembre del 2018)

Así, los funcionarios pretenden posicionar socialmente a las víctimas dentro de estatus "mejores" o incorporarlos dentro de estilos de vida "ideales" como es el caso de la vida que afronta la población de Cajicá ajena al conflicto armado, es decir, la población civil que no ha vivido directamente las consecuencias del conflicto armado. Incluso a través de todas estas representaciones se puede ver que intentan alivianar el impacto del conflicto transformando los imaginarios que los marginalizan, controlar los casos de revictimización, así como fortalecer su autoestima para evitar su discriminación o cualquier sentimiento de menosprecio.

Además de existir una relación entre los funcionarios, la población civil y las víctimas aun desde la consideración de vulnerado, como se evidencio anteriormente, es digno de señalar que en la asistencia que se brinda a las víctimas (por lo menos en el caso de Cajicá) se ha desdibujado que existen poblaciones con necesidades diferentes y más apremiantes unas que otras.

Conforme a las circunstancias, todas las víctimas han sufrido las consecuencias del conflicto armado, pero se encuentran en una posición socioeconómica diferente, algunas están estables o cuentan con más redes de apoyo. En las entrevistas realizadas a un secretario municipal (primer caso), y la Personería (segundo caso) se puede observar que no todas las víctimas se encuentran en situación de igualdad, como tampoco todas requieren de una intervención similar, de allí la importancia de hacer una caracterización para llevar a cabo un efectivo goce de derechos. Para el primer caso:

vienen de otros pueblos, pero están trabajando acá, entonces si trabajan viven, entonces hay medios yo no les puedo entregar ninguno de los subsidios. Lo que te dije, cuando uno hace la visita ve la necesidad automáticamente, si les damos a todas las personas del conflicto armado o víctimas cualquiera de estos beneficios, porque cada uno tiene una cantidad diferente de necesidades, no lograríamos priorizar a los que de pronto si lo necesiten. (Secretario, entrevista 28 de diciembre del 2018)

En cuanto al segundo caso:

la Personería, en el marco de la semana por la paz en septiembre, dio una charla a todos los servidores públicos sobre lo que era la Ley 1448, precisamente para que los servidores públicos entendieran cuál es el significado del concepto de víctima, [...] y recordándoles que el imaginario de la población víctima no es el desplazado que vemos nosotros en los semáforos o en la calle, sino que hay po- blación víctima como un Alan Jara qué fue gobernador del Meta y, a pesar de esto él tiene el estatus de víctima, por ejemplo, o sea, hay población víctima de todos los órdenes. (Personería Municipal de Cajicá, entrevista, 14 de diciembre de 2018)

Teniendo en cuenta las condiciones en que se encuentran las víctimas, la asistencia se desarrolla atribuyéndole un rol activo al Estado y, por ende, a los funcionarios que adquieren compromisos en el marco de lo planteado en la Ley 1448 de 2011. De forma que, del otro lado, las víctimas pueden constituirse en sujetos pasivos. En lo tocante a la pasividad de las víctimas, vale la pena señalar que es una característica que posiblemente desde el mismo Estado se ha venido implementando. De ahí que, ningún funcionario entrevistado haya mencionado planificar una intervención en la que se considere la participación de las mismas víctimas que tienen una interpretación de su propia situación y, posiblemente, unas iniciativas desde las cuales dar soluciones. Para poner en palabras concretas esta situación, a continuación la entrevista a la Personería Municipal:

ha sido mal enfocado el manejo de la población víctima porque de pronto desde un comienzo debió haberse dirigido a que ellos realmente estabilizarán sus proyectos de vida y empezarán a reincorporarse en una sociedad productiva y se les dio al principio un enfoque muy asistencial y ya como que la población víctima espera solamente asistencialismo. (Personería Municipal de Cajicá, entrevista, 14 de diciembre de 2018)

Hipótesis 2. Desde la acción operativa de asistencia que realizan los actores que tienen alguna responsabilidad con las víctimas se ofertan ayudas generalizadas para los vulnerables.

Dentro del Plan de desarrollo municipal "Cajicá nuestro compromiso" 2016-2019, existe el objetivo de reducir la brecha de pobreza incluyendo acciones tanto para las familias en condición de vulnerabilidad como para las víctimas del conflicto armado. No obstante, pese a que existen diferentes indicadores para estos dos grupos, según lo escuchado a las personas que se entrevistaron, las acciones se generalizan para ambos grupos.

Aunque se confirme través de las entrevistas que existe una oferta general, en el Plan de desarrollo se encuentra un apartado titulado como "diagnostico", dentro del cual se exponen específicamente los diferentes programas en los que priorizan la participación de las víctimas (Concejo Municipal de Cajicá, 2016, p. 228).

Asimismo, se exponen los desafíos frente a las limitaciones de la asistencia. En cuanto a este último aspecto, vale la penar resaltar que Cajicá no cuenta con una caracterización tal como lo requiere (artículo 172 de la 
Ley 1448 de 2011) en el sentido de agrupar de acuerdo con las necesidades específicas e insatisfechas de la población de víctimas del conflicto armado, a través de lo cual se canalice una oferta específica. Si bien se tiene conocimiento acerca de la necesidad de caracterizar desde el 2016, fecha de difusión del Plan de desarrollo de Cajicá, en la fecha 2018 de realización de este estudio los funcionarios continúan evidenciando esta problemática como una dificultad. Por esta razón, en la entrevista a la personería se enuncia:

la Personería ha dejado constancia, tanto en la mesa municipal de participación de víctimas como en el Comité Municipal Territorial de Justicia Transicional sobre la necesidad de la caracterización, de que se debe saber con exactitud cuáles son las necesidades de la población víctima que tiene el municipio, porque incluso las cifras son diferentes la que tiene el enlace municipal, la que tiene la personería, la que tiene el consejo municipal, la que tiene la misma nación. (Personería municipal de Cajicá, entrevistas, 14 de diciembre de 2018)

Continuando con la idea del personero municipal en lo relativo a la falta de un estudio que caracterice la población de víctimas que habitan en el territorio, en el Plan de desarrollo municipal específicamente se dice lo siguiente: "Las estadísticas que se reportan a nivel nacional no coinciden con los datos que se tienen a nivel municipio, por lo cual se toma como base, los datos reportados por el SISBEN" (Concejo Municipal de Cajicá, 2016, p. 230).

Aunque el problema de caracterización influye en la necesidad de hacer una oferta especifica que motive la participación de la población víctima, la cual verá en dicha oferta una solución a sus necesidades insatisfechas, se observa que algunos funcionarios tienden a responsabilizar a las víctimas de no aprovechar o no acceder a las ofertas existentes. Así pues, se enuncia que:

en el Comité de justicia transicional hemos evidenciado que de toda la oferta institucional y de todas las acciones administrativas que tenemos en este momento implementadas hay muchas que la población no está aprovechando, no se está aprovechando todo lo que el municipio está ofreciendo para esta población. (Secretario, entrevista, diciembre 13 de 2018)

Hipótesis 3. En escenarios de diálogo no existe participación suficiente y activa que represente los intereses de las víctimas.

De igual manera, el funcionario que hace de enlace municipal de víctimas justifica la generalización de la oferta debido a la poca información que se puede obtener de las víctimas, lo anterior a raíz de la escaza participación dentro de los escenarios en los que se convocan:
No, no lo hemos hecho de manera específica porque, como te digo, en los muchos escenarios de participación a los que se les ha invitado no hemos tenido la suficiente participación de las víctimas, lo que quiere decir que, en teoría, nos da como temor desgastarnos en un tema, entonces por eso la oferta generalmente siempre está centralizada en cada una de las secretarías y se espera o se canaliza la oferta desde el enlace municipal hacia las secretarías. (Enlace a municipal de víctimas, entrevista, 11 de diciembre del 2018)

Como se ve, esto se convierte en un círculo donde las víctimas se sienten insatisfechas con las medidas o soluciones que reciben del Estado, desconocen cómo participar en estos escenarios y, asimismo, desconocen cuál es su rol en la defensa de derechos como lo son exigir las ofertas para cubrir sus necesidades insatisfechas acorde con lo orienta el enfoque diferencial en la Ley 1448 de 2011.

Hipótesis 4. Las ofertas para brindar atención a las víctimas desde el sector público o privado se desarrollan de forma desarticulada.

Con todo esto se puede asegurar que, dentro del municipio de Cajicá, no existe intervención en lo concerniente al enfoque diferencial más allá de ser una propuesta en la que se incorpora a la víctima o, más bien, a la persona vulnerable dentro de las diferentes agendas de intervención que tienen las instituciones dentro del territorio. En consecuencia, por un lado, llega Secretaría de Educación brindando una oferta específica en el ámbito de su competencia; por otro lado, se acerca el Ministerio de Salud proporcionando una oferta en el ámbito de su facultad, y así sucesivamente cada actor que tiene responsabilidades con las víctimas llega a Cajicá interviniendo desde diferentes frentes sin articularse ni dialogar con los otros actores para ofertar una propuesta integral, tal como se evidencia en la siguiente afirmación:

cada oferta específica está en cabeza de una Secretaría que debe garantizar el acceso a ese tipo de oferta. Si es de nivel educativo se hace responsable a la Secretaría de Educación, si es de nivel de emprendimiento y empleabilidad depende de la Secretaría de Desarrollo Económico, si es un tema de vivienda depende pues de Vivienda, si es un tema de deportes depende de Deportes, si es cultura depende del Instituto de Cultura, dependiendo cuál es la oferta que pide la familia. (Enlace municipal de víctimas, entrevista, 11 de diciembre de 2018)

Acerca de la articulación territorial (municipio) con los sectores privados, es valioso mostrar que, aunque existen ofertas de trabajo y programas para las víctimas dentro Cajicá, aún es necesario, por un lado, planear más acciones y espacios de integración para este tipo de población 
y, por otro lado, destinar un porcentaje del presupuesto de las empresas privadas, de forma que se pueda compensar las insuficiencias presupuestales del ente territorial con el tema de las víctimas. En conclusión, aún hace falta una mejor articulación entre las empresas privadas que puede atender a las víctimas y el ente territorial.

Hipótesis 4.1. No existe articulación para brindar una oferta integral a las víctimas ni se tiene en cuenta la posibilidad de implementar la articulación horizontal y vertical

A continuación, los conceptos de articulación vertical y horizontal sirven para ilustrar las posibilidades que tienen las instituciones para organizar la oferta en las políticas públicas y garantizar una gestión eficiente y eficaz. Según Licha y Molina (2006), la articulación horizontal propende por la conexión entre las entidades del mismo nivel: nacional o territorial y la articulación vertical propende por la conexión entre la nación y el territorio.

Frente a estos dos tipos de articulación es posible triangular los resultados obtenidos de esta investigación con los obtenidos en la investigación titulada Evaluación de operaciones y de resultados de la política de Retornos y Reubicación para el Departamento Nacional de Planeación (DNP) durante el 2017. Los resultados obtenidos en dicha evaluación son producto de la interrelación con procesos de retorno y reubicación experimentados en 34 municipios del país. En términos del presente artículo, es significativo comparar nuestros resultados con los hallazgos del anterior macroestudio, considerando que así se puede visualizar desde una perspectiva más amplia el problema de articulación institucional.

Como ya se enunció, la percepción evidenciada en el municipio de Cajicá refiere a que la oferta de programas hacia la población de víctimas se hace de manera individual y no se articulan los oferentes para lograr una intervención sobre la población de víctimas, a pesar de lo que se oriente en la Ley 1448 de 2011.

Este resultado es muy semejante a lo obtenido en el estudio anteriormente citado, el cual determina respecto a la percepción que tienen los funcionarios de la gestión en cuanto a la articulación que el promedio de calificación para actores nacionales o territoriales no supera el 3.1 en una escala de 1 a 5 . Lo anterior significa la poca implementación de trabajo en equipo para dar solución a necesidades insatisfechas de sectores vulnerables y vulnerados de la población, por lo que implica una doble inversión de recursos, de esfuerzos, para cubrir una misma y determinada necesidad. La Figura 2 ilustra los resultados obtenidos en dicho estudio.

Desde luego, el anterior aspecto podría estar evidenciando en todo el contexto nacional, tal como lo hace el estudio al que se refiere la Figura 2 y que se corrobora en el presente estudio realizado para Cajicá, una problemática que no solo apunta a evidenciar un hecho de ocurrencia nacional, sino que podría estar conectado con otros hechos estructurales en la administración pública de nuestro país.

De este modo, un funcionario del municipio de Cajicá percibe la articulación como se menciona a continuación:

me parece muy importante establecer cómo se está llevando a cabo lo que debería ser la conexión entre el ente territorial, el departamento y la nación, donde señalo que hay una gran responsabilidad de la nación en el tema de víctimas porque allí está presupuesto y allí están las funciones, ahí están las líneas, ahí están las políticas públicas. (Personería, entrevista, 14 de diciembre de 2018)

Si bien la desarticulación es un problema que afecta la eficiencia de la política pública, el funcionario continúa desarrollando su idea frente a este aspecto, diciendo lo siguiente:

esto tiene tres eslabones: el nivel nacional, el nivel departamental y el ente territorial. Lo que está haciendo el municipio es lo que le corresponde, lo que hace la Personería es lo que le corresponde, pero encontramos una

\begin{tabular}{|c|c|c|c|c|c|c|}
\hline \multirow[t]{2}{*}{ PREGUNTA } & \multicolumn{3}{|c|}{$\begin{array}{l}\text { CALIFICACIÓN ACTORES } \\
\text { NACIONALES }\end{array}$} & \multicolumn{3}{|c|}{$\begin{array}{l}\text { CALIFICACION ACTORES } \\
\text { TERRITORIALES }\end{array}$} \\
\hline & PROMEDIO & MINIMO & MAXIMO & PROMEDIO & MiNIMO & MÁXIMO \\
\hline $\begin{array}{l}\text { De } 1 \text { a } 5 \text {, siendo } 5 \text { la calficación más alta, ¿Qué tan } \\
\text { articuladas están las entidades del SNARIV en el } \\
\text { nivel nacional para implementar los procesos de } \\
\text { retorno y reubicaciones? }\end{array}$ & 2,3 & 1 & 4 & 2,3 & 1 & 5 \\
\hline $\begin{array}{l}\text { De } 1 \text { a } 5 \text {, siendo } 5 \text { la calificación más alta, ¿qué tan } \\
\text { articuladas están las entidades del SNARIV en el } \\
\text { nivel territorial para implementar los procesos de } \\
\text { retorno y reubicaciones? }\end{array}$ & 2.5 & 1 & 4 & 3,1 & 1 & 5 \\
\hline $\begin{array}{l}\text { De } 1 \text { a } 5 \text {, siendo } 5 \text { la calificación más alta, ¿Qué } \\
\text { tan articuladas están las entidades del SNARIV } \\
\text { entre el nivel nacional y el territorial para } \\
\text { implementar los procesos de retorno y } \\
\text { reubicaciones? }\end{array}$ & 1,9 & 1 & 3 & 2,4 & 1 & 5 \\
\hline
\end{tabular}

Figura 2. Calificación de actores nacionales y territoriales a la articulación para implementar los procesos de RR

Fuente: DNP (2017a, p. 58). 
desconexión con el departamento y con la nación, entonces te voy a poner un ejemplo concreto: llega en este momento un ciudadano que dice que es víctima y que el busca que se le reconozca como víctima, la personería debe recibirle la declaración para establecer, esto tienen un unos protocolos y tiene un formato que está establecido en una forma de la Unidad de Víctimas incluso en línea, nosotros le recibimos la información es una declaración que él da, entonces se establece precisamente cuál fue la los hechos por los cuales él tuvo que salir de su sitio de vivienda, lo que sea y que todos los hechos que lo hacen víctima, eso lo recibe hoy la Personería, en línea se lo envía la Unidad de Víctimas y la Unidad de Víctimas le está respondiendo a esa persona si es o no es víctima en un término aproximado de cuatro a seis meses, entonces la Personería oportunamente recibe la declaración, la recepciona, hace su trabajo, pero arriba no hay la misma celeridad, la misma agilidad para responderle a ese ciudadano. Si usted es víctima o no es víctima y tienen deambulando al ciudadano cuatro a seis meses a ver si sí o si no, y lo mismo sucede con otro tipo de situaciones, donde el ciudadano tiene que entutelar y nos llegan en las respuestas a nosotros para entregárselas a esa población víctima, entonces en este momento uno encuentra que el municipio empieza a navegar con la población víctima. (Personería Municipal, entrevista, 14 de diciembre de 2018)

Frente a la opinión del funcionario, se puede decir que, así como sucede en el panorama local, dentro del municipio de Cajicá existe una profunda problemática de desarticulación, la cual se evidencia, por un lado, en el vacío institucional existente en la aplicabilidad de la normatividad para proveer una asistencia que se adapte a las condiciones sugeridas en la ley y, por otro lado, en una asistencia que satisfaga las expectativas de las necesidades insatisfechas de las víctimas. Por esta razón, la asistencia subordina a las víctimas a diferentes soluciones o posibilidades que, en términos de sus necesidades, son ineficientes, reiterativas y poco consultadas en el caso de las comunidades étnicas desde sus habilidades o para su sostenibilidad. Teniendo en cuenta todos estos elementos, resulta indudable decir que el enfoque diferencial no es transversal a la asistencia o a las ofertas.

Aparte de que el problema de articulación afecta la integralidad de la asistencia, hay que sumar otro factor como lo es la escaza asignación presupuestal proporcionada a los municipios para atender las necesidades de las víctimas. Este aspecto no solo surge durante el trabajo de campo realizado en Cajicá (primer caso), sino que también se puede ver en el trabajo que realizo la firma Econometría para el DNP:

ahora la nación debería ayudar a los municipios con recursos en estos proyectos productivos tal cual como lo hace el departamento el SIPOT de la gobernación, lo que pasa es que son limitados los recursos son limitados, entonces se debería sacar ayudarnos, si bien es cierto nos ayudan con esa digamos oferta nacional como el SENA para los cursos que hemos hecho por ejemplo las niñas de confección estuvieron con el SENA, pero la hora de tener recursos quien está poniendo los recursos en un momento dado el municipio, no hay una ayuda de recurso nacional que me diga oiga yo voy a gestionar algún proyecto con la nación en donde yo pueda ayudar a un grupo de personas que tuvieron esa problemática. (DNP, 2017a)

Asimismo, aludiendo a los resultados de la investigación externa, en una entrevista a la Defensoría del pueblo, un funcionario identifica que "a pesar de la reducida asignación presupuestal que se observa en algunos municipios para atender a sus víctimas, los gobiernos locales no están haciendo ninguna gestión de recursos a sus respectivas gobernaciones ni de estas al nivel nacional" (DPN, 2017a, p. 59).

Hipótesis 5. Entre los discursos que manejan los actores que tienen alguna responsabilidad con las víctimas, existe una diversidad de opiniones en relación con lo que representa el enfoque diferencial, de manera que cada quien termina realizando su interpretación y desenfocándose de los parámetros que consagra la Ley 1448 del 2011.

Concretamente, cuando se les pregunta a los funcionarios sobre la comprensión del enfoque diferencial, existen diferentes percepciones. Por ejemplo, se hallan respuestas enmarcadas en el cumplimiento de lo establecido en la Ley 1448 de 2011:

dando cumplimiento a la Ley 1448 de 2011, digamos que su enfoque de política pública tiene como priorización la prevención, protección, garantías de no repetición de las víctimas, la asistencia y la atención a las mismas con enfoque de derechos y tiene el tema de reparación integral. (Enlace municipal de víctimas, entrevista, diciembre 11 de 2018)

Se alude a que debe tenerse presente específicamente a la población indígena, afrodescendiente y ROM. Al respecto, valga la aclaración de que, para el funcionario entrevistado, no se puede aplicar el enfoque diferencial dada la ausencia de esta población en Cajicá:

en este momento no podríamos decir que se pueda ver el enfoque diferencial, porque el enfoque diferencial como decía está hacia las indígenas, nosotros no tenemos población indígena reconocida que la tengamos detectada; a la población afrodescendiente donde tampoco hay una organización de afrodescendientes. (Personería Municipal de Cajicá, entrevista, diciembre de 2018)

Por otro lado, se enuncia este enfoque como un planteamiento discriminatorio: 
pienso de manera personal que el enfoque diferencial a veces en lo que se está convirtiendo es en un enfoque discriminatorio y muchas víctimas por ejemplo que es el caso puntual se sienten re victimizadas al identificarse como víctimas o al pedirse que se acerquen a programas que son específicamente para ellos. (Secretaría, entrevista, diciembre de 2018)

En conclusión, se evidencia que cada funcionario desde su frente, es decir, desde su agenda, asume una comprensión diferente de lo que cree representa el enfoque diferencial, contradiciendo los objetivos que se propone la Ley 1448 de 2011.

En atención a lo expuesto, es relevante enlazar este análisis con los resultados obtenidos en estudios de investigación externos con coberturas más amplias en relación con el número de actores que participan en la ejecución de la política pública, como lo es: "Evaluación de la política pública para la prevención de riesgos, la protección y garantía de los derechos de las mujeres víctimas del conflicto armado" (Conpes 3784 de 2013), realizada en el año 2017 por la consultoría Econometría para el Departamento Nacional de Planeación. En este se trabajó con tres metodologías diferentes: mapeo de actores, scorecard $\mathrm{y}$ entrevistas a organizaciones de mujeres, organismos de cooperación internacional y a funcionarios del orden nacional y territorial. La consultoría indagó acerca de la claridad de aplicación del enfoque diferencial desagregado por género obteniendo los siguientes resultados que será importantes triangular con los resultados obtenidos en nuestro estudio (Figura 3).

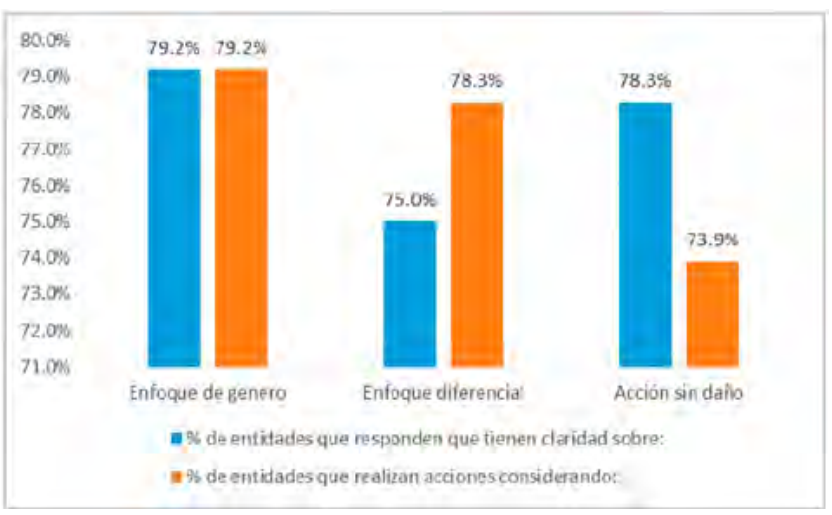

Figura 3. Percepción de las entidades nacionales de la incorporación de enfoques en sus políticas de atención a mujeres víctimas.

Fuente: DPN (2017b, p. 52).

De acuerdo con el autor del estudio del Conpes 3784, es preciso decir que la Figura 3 muestra cómo se tiene comprensión y se realizan acciones específicas en el enfoque de género. En el caso del enfoque diferencial manifiestan tener poco conocimiento, aunque consideran que realizan acciones en relación a este enfoque.

En relación con el tema del conocimiento que tendrían los actores oficiales sobre el enfoque diferencial, específicamente la consultoría encontró que existe una diversidad de interpretaciones en la medida que los equipos al interior de las entidades han desarrollado su propia conceptualización (DPN, 2017b, p. 52). En este aspecto hay total coincidencia con los resultados expuestos anteriormente y se interpreta de igual forma en las entrevistas realizadas por esta investigación en Cajicá.

\section{Conclusiones}

En lo que concierne a la evaluación realista, se puede decir que este enfoque contribuye al conocimiento de un fenómeno de forma más profunda, ya que en la medida que el investigador va ejecutando una serie de hipótesis $\mathrm{y}$, por ende, reformulando o mejorando otras, se va indagando más profundamente el fenómeno. Al contrario de la investigación tradicional, la ER da cabida a que se indague por categorías emergentes que van surgiendo en la medida en que se van contrastando nuevas hipótesis, lo que permite acercar el lente para delimitar la funcionalidad de una política pública y evidenciar la efectividad, el logro de objetivos y los niveles de impacto. De la misma manera, la aplicación de la teoría realista y sus resultados podrían considerarse como enunciados producto de investigación inductiva o enunciados construidos a través de percepciones, discursos que, al ser triangulados, bien pueden ratificar, complementar o falsear enunciados teóricos o estructurar nuevos argumentos. De esta manera, es una herramienta útil que bien podría utilizarse dentro de los mecanismos para trabajar teoría fundamentada.

En lo relativo al enfoque diferencial, aunque existe un concepto general estipulado por la Ley 1448 de 2011, este concepto suele tener múltiples interpretaciones entre los funcionarios que deben aplicar la ley en cada una de las instituciones encargadas de atender la problemática de las víctimas. De tal manera, al no existir una caracterización de la población como lo orienta la ley, el resultado es la inclusión de la población de víctimas dentro de la oferta general de intervención social que hace el municipio. Lo anterior puede llegar a determinar que la población víctima no reciba la totalidad de la oferta, que las ofertas sean entendidas como asistencialismo y no como respuesta a las necesidades insatisfechas de dicha población. Se considera entonces, una asistencia a través de la cual, las víctimas que han sufrido los estragos de la guerra, y han sido vulnerados de forma particular por razón de edad, género, orientación sexual y situación de discapacidad no pueden ser reparados y acceder a la justicia de manera equitativa, sino que son parte de una precaria, desarticulada y competitiva estrategia para acceder a los programas del gobierno. 
Por otro lado, la desarticulación entre instituciones es un elemento que contribuye a que se sigan proporcionando ofertas de poco impacto. Sería conveniente la existencia de una relación articulada entre las instituciones, tanto en el sentido vertical como horizontal, ya que así se podría llegar a mejorar el modelo de asistencia que reciben las víctimas.

Pensar en la transformación de la forma en que se oferta y lo que se oferta posiblemente involucra (por ejemplo, en el contexto especifico de Cajicá) el fortalecimiento y la participación de las víctimas dentro de los escenarios de diálogo y de toma de decisiones conjuntamente con los actores que representan al Estado. En términos concretos, esto puede llegar a significar la necesidad de fortalecer o consolidar las organizaciones sociales para una debida participación en la ejecución de políticas públicas dentro del marco de un territorio o en poblaciones específicas.

\section{Referencias}

Centro Nacional de Memoria Histórica [CNMH] (2013). jBasta ya! Memorias de guerra y dignidad.

Concejo Municipal de Cajicá (2016). Acuerdo 04 de 2016 Plan de desarrollo 2016-2019. "Cajicá, nuestro compromiso". https:// sigot.igac.gov.co/sites/sigot.igac.gov.co/files/acuerdo_004_ de_2016_plan_de_desarrollo_2016-2019-2_0.pdf

Congreso de Colombia (2011). Ley 1448 de 2011. https://www.unidadvictimas.gov.co/sites/default/files/documentosbiblioteca/ley1448-de-2011.pdf
Consejo Nacional de Política Económica y Social [Conpes]. (2013). Conpes 3784 de 2013.

Departamento Nacional de Planeación [DNP] (2017a). Evaluación de operaciones y resultados de la política de retornos y reubicación. https://colaboracion.dnp.gov.co/CDT/Sinergia/Documentos/ Evaluacion_politica_retornos_reubicaciones_Documento.pdf

Departamento Nacional de Planeación [DNP] (2017b). Evaluación de la política pública para la prevención de riesgos, la protección y garantía de los derechos de las mujeres víctimas del conflicto armado (Conpes 3784 de 2013). http://www.equidadmujer.gov.co/oag/Documents/Evaluacion-Mujeres-Victimas-2017-CONPES_3784.pdf

Jagosh, J., Bush, P., Salsberg, J., Macaulay, A., Greenhalgh, T. y Wong, G. (2015). A realist evaluation of community-based participatory research: partnership synergy, trust buikding and related ripple effects. BMC Public Health, 15.

Licha, I. y Molina, C. (2006). Coordinación de la política social: criterios para avanzar. Instituto Interamericano para el Desarrollo Social.

Navarro, H. (2005). Manual para la evaluación de impacto de proyectos y programas de lucha contra la pobreza. Cepal.

Parra, J. (2017). Introducción a la evaluación realista y sus métodos: ¿qué funciona, para quién, en qué aspectos, hasta qué punto, en qué contextos y cómo? Economía y región, 11(2), 11-44.

Pawson, R. y Tilley, N. (1997). Realistic Evaluation. Sage.

Westhorp, G. (2014). Realist Impact Evaluation. Overseas Development Institute. 